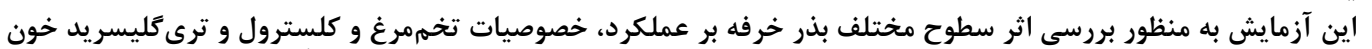

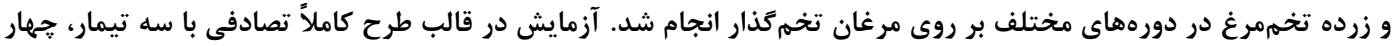

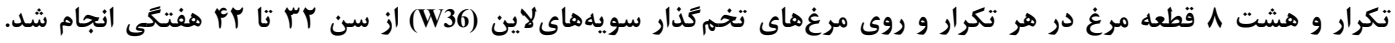

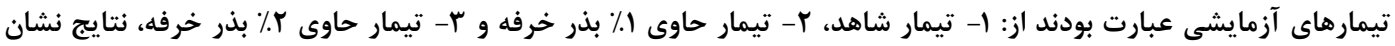

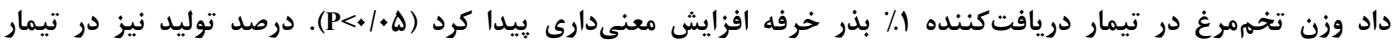

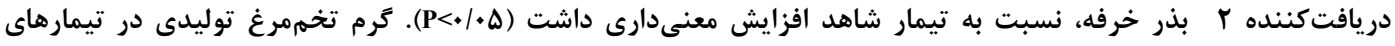

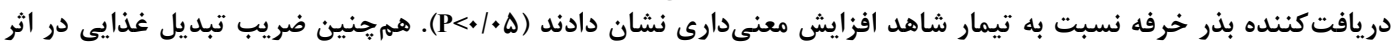

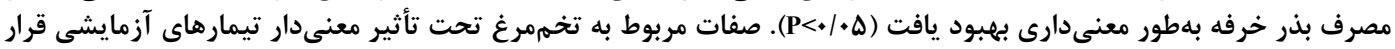

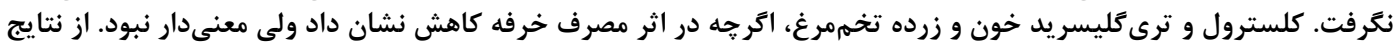

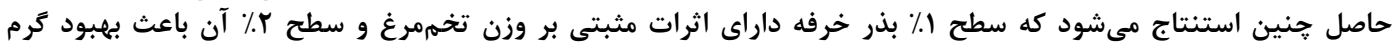

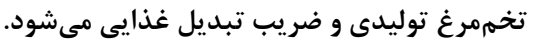

وازههاى كليدى: مرغان تخمكذار، بذر خرفه، عملكرد، خصوصيات تخممرغ، كلسترول زرده

آنتى اكسيدان و عناصر معدنى متعدد شامل: آهن، مس،

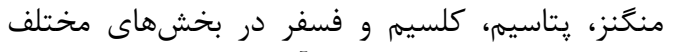

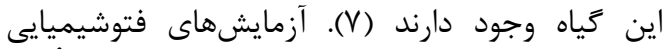

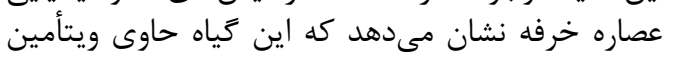

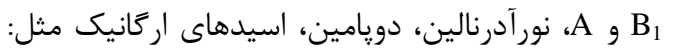

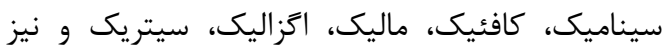

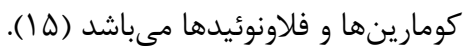

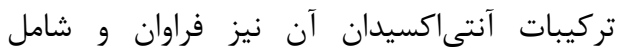

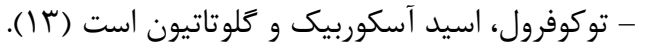

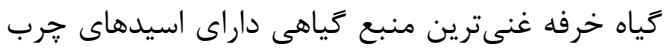

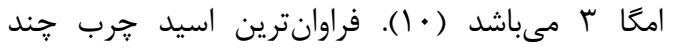

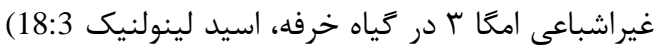

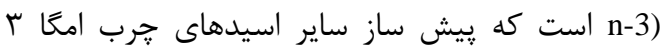

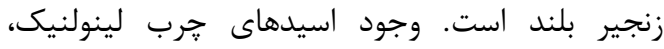

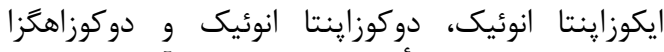

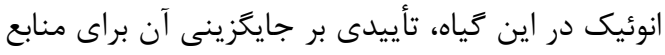

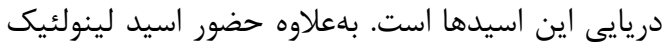

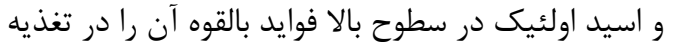

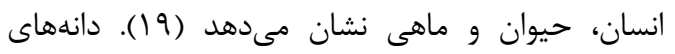

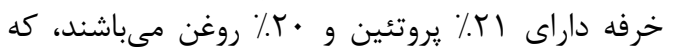

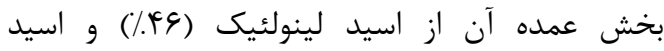

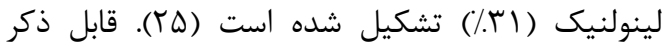
است كه هيج نشانه سمى قابل توجهى هنوز در ارتباط با لثا
به علت پيشرفت علم تغذيه و آحاهى مردم به ارزش

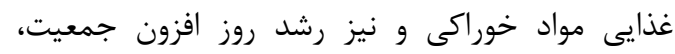

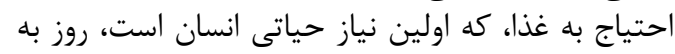

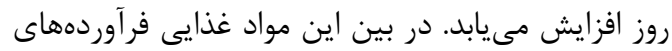

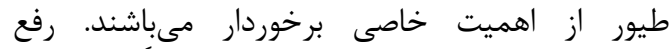
كمبودهاى غذايى در جوامع بشرى، مخصوصاً كشورهايى

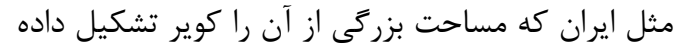

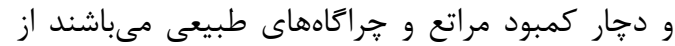

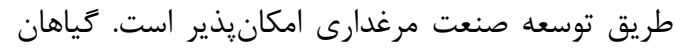

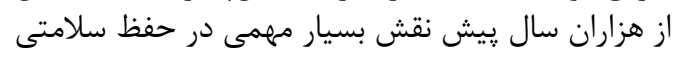

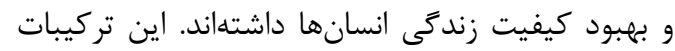

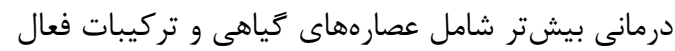

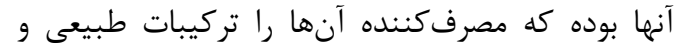

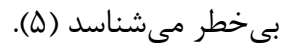

خرفه (Purslane) با نام علمى Portulaca Oleracea) گياهى است علفى، يكساله با ساقهاى گتوشتدار و و

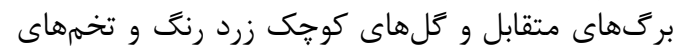

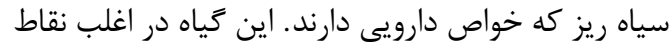

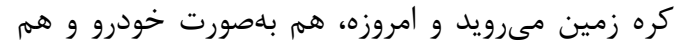

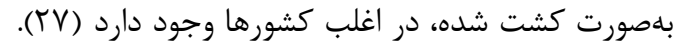

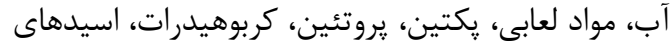

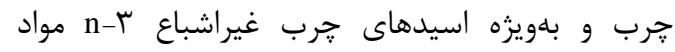


شد. در يايان هر دوره آزمايشى (جهار هفته) از هر

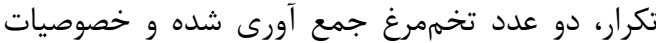

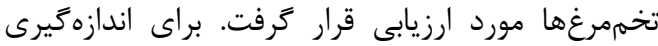

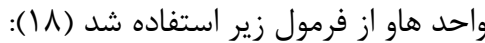

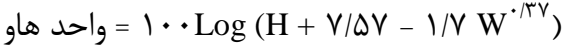

كه در اين فرمول H عبارت است از ارتفاع سفيده غليظ

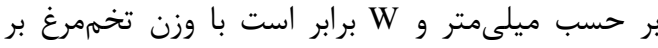

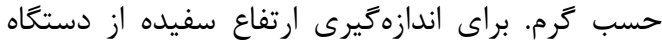

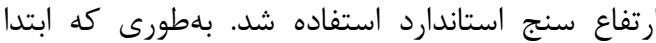

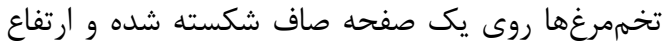

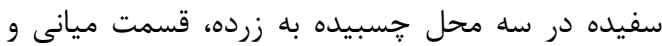

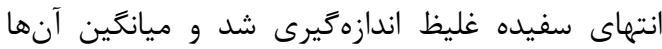

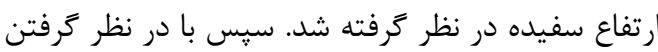

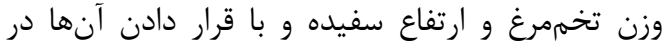

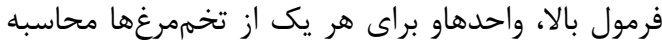

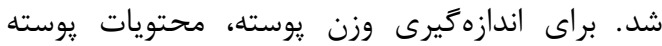

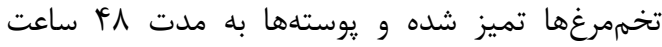

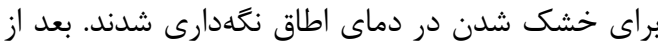

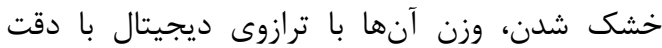

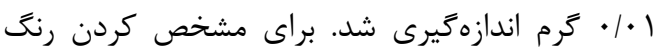

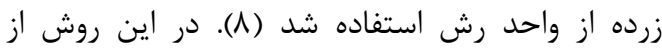

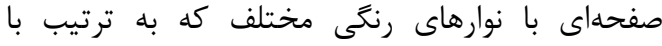

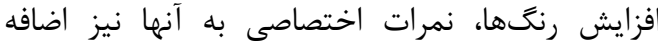

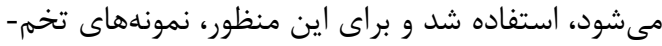

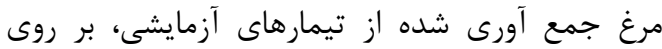

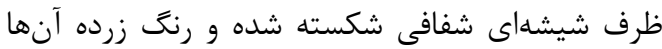

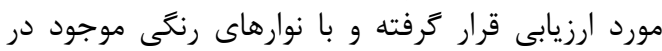

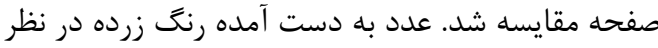

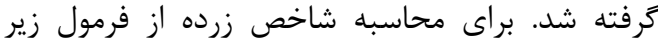

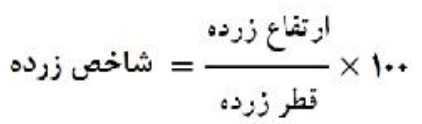

استفاده شد (9):

براى اندازمخيرى ميزان كلسترول و ترى تركليسريد

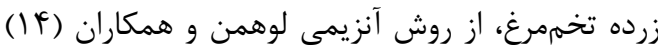

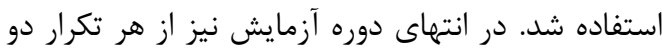

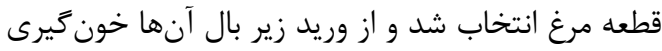

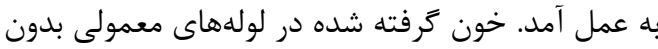

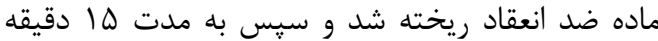

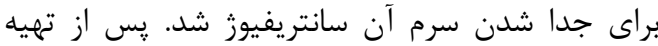

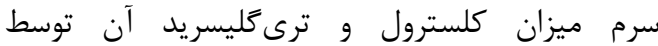

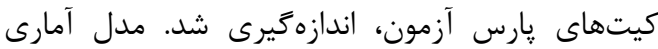

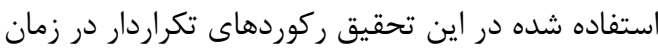
بود. دادههاى بدست آمده (Repeated Measurement)

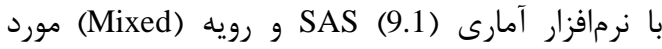

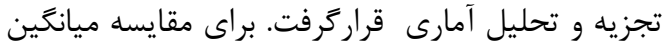
تيمارها از آزمون توكى كرامر استفاده شد.
اين كياه گزارش نشده است (9). از آنجايى كه نتايج

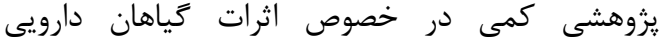

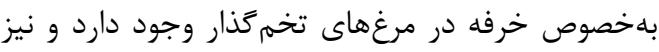

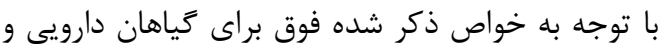

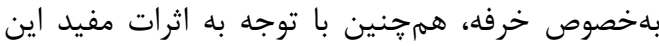

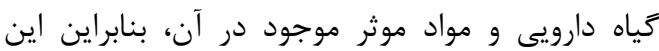

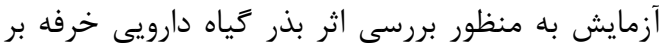

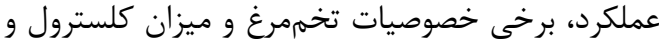

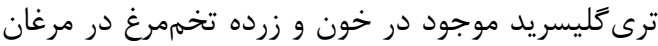

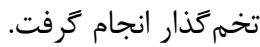

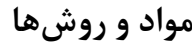

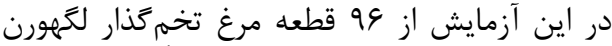

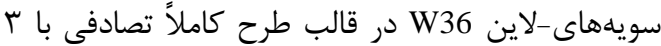

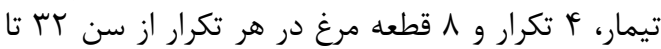

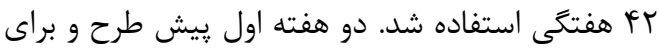

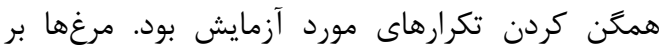

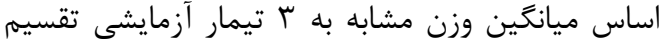

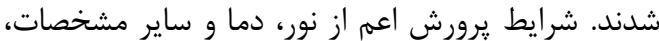

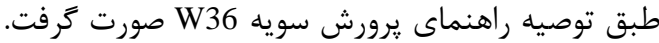

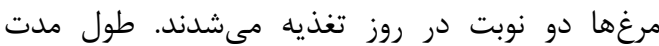

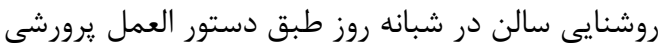

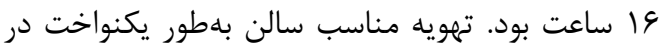

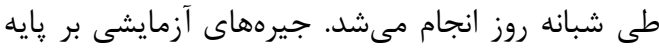

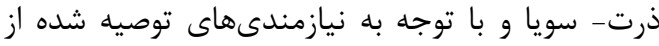

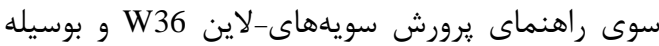

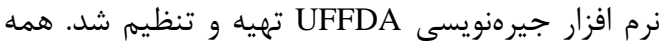

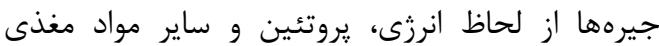

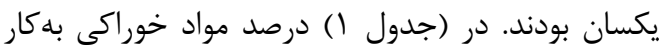
رفته براى تهيه جيرههاى آزمايشى و مواد داد مغذى تأدي تأمين

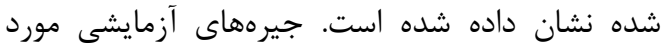

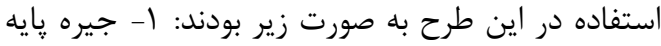

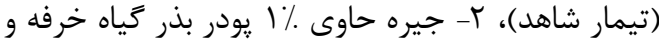

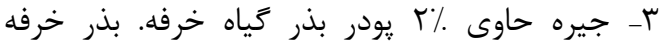

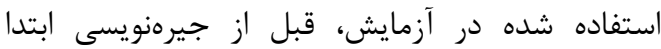

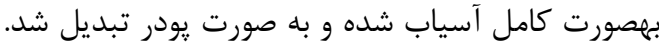

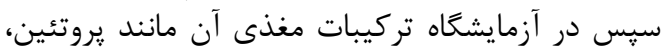

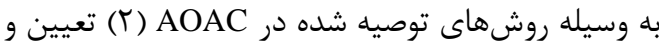

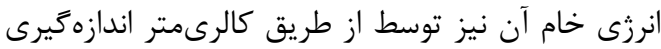

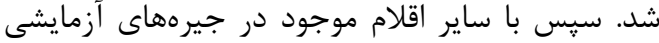

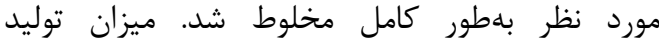

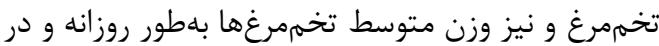

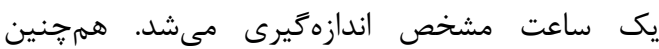

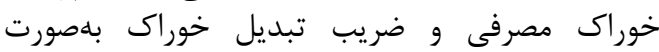

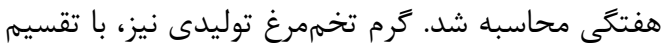

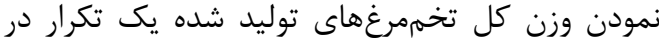

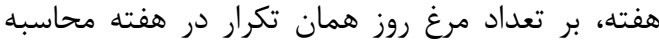




\begin{tabular}{|c|c|c|c|}
\hline \multicolumn{4}{|c|}{ جدول 1 - تركيب جيرههاى آزمايش و مواد مغذى تأمين شده از طريق آنها } \\
\hline$\Delta G / V I$ & $\Delta \mathrm{V} / \mathrm{V} r$ & $\Delta \Lambda / V \Delta$ & ذرت \\
\hline$r \Delta / T V$ & $r \Delta / \uparrow \wedge$ & $r \Delta / v \cdot$ & كنجاله سويا \\
\hline$r / V F$ & $r / \Delta r$ & r/Tr & روغن \\
\hline$\Delta / .9$ & $\Delta / \cdot 9$ & $\Delta / \cdot V$ & يوسته صدف \\
\hline$r /$. & $r /$. & $r /$. & سنع "هَ آهى \\
\hline$r / 1 \Delta$ & t/lF & r/lr & دى كلسيم فسفات \\
\hline$\cdot / \Delta$ & $\cdot / \Delta$ & $\cdot / \Delta$ & مكمل ويتأمينى و مواد معدنى" \\
\hline$r / \cdot \cdot$ & $1 / \cdot$ & $\cdot 1 \cdot$ & 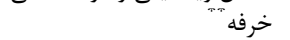 \\
\hline$\cdot / \mu$ & $\cdot / \mu$ & $\cdot / \mu$. & نمكى \\
\hline שT/ & $\cdot / 4 t$ & $\cdot|r|$ & متيونين \\
\hline \multirow[t]{2}{*}{$\cdot 1 \cdot 4$} & . & $.1 \cdot \mathrm{r}$ & ليزين \\
\hline & & & مواد مغذى تأمين شده \\
\hline rAf. & rAY. & rAY. & انرزى (كيلو كالرى/ كيلوكرم) \\
\hline $19 / \pi$. & $19 / \pi$. & $19 / \pi$. & 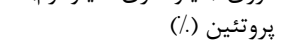 \\
\hline$\varphi /$. & $\varphi /$. & $\varphi /$. & كلسيم (.) \\
\hline$\cdot / \Delta$ & $\cdot / \Delta$ & $\cdot / \Delta$ & فسفر (./) \\
\hline$\cdot / 4 V$ & $\cdot / 4 V$ & $\cdot / 4 V$ & متيونين (.) \\
\hline$\cdot / 19$ & $\cdot / 19$ & $\cdot / 149$ & لايزين \% \\
\hline$\cdot / V \Delta$ & $\cdot / V \Delta$ & $\cdot / V Q$ & متيونين- سيستئين (./) \\
\hline .19 &.$/ 9$. & .19 & ترئونين (//) \\
\hline . & . & - Rt & تريتيتوفان (./) \\
\hline$\cdot / K F$ & $\cdot / K F$ & $\cdot / \pi F$ & سديم (./) س س (س) \\
\hline \multicolumn{4}{|c|}{ 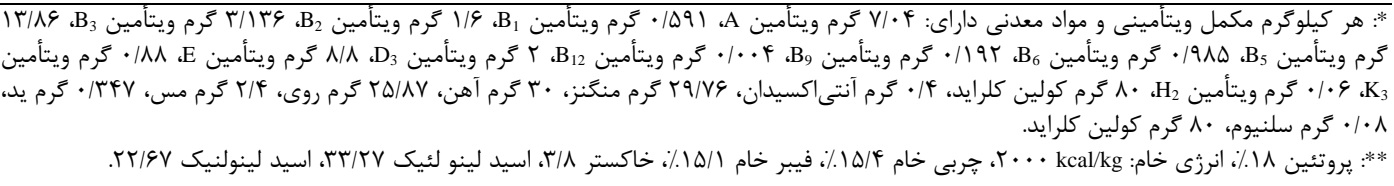 } \\
\hline
\end{tabular}

در هيج كدام از دورههاى آزمايش، تحت تأثير معنى دار

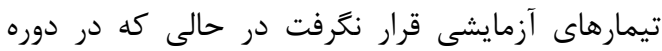

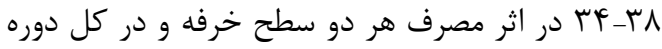

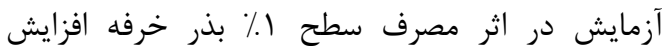

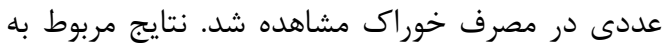

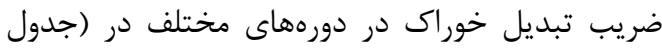

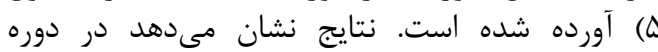
كاهب-r

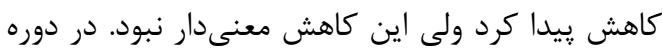

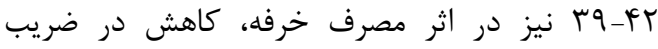

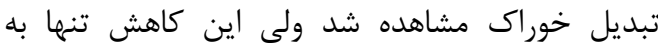

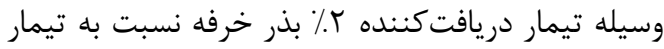

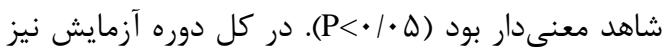

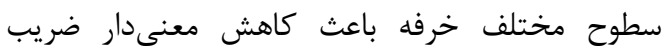

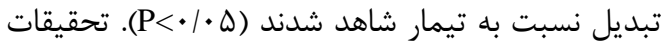

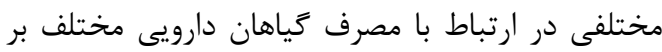

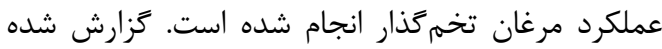

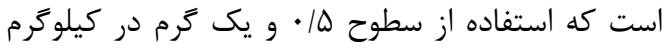

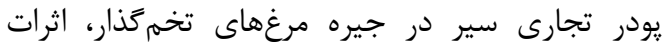

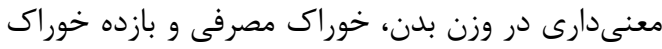

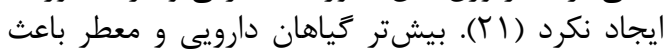

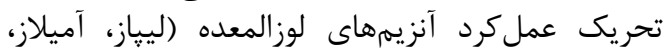

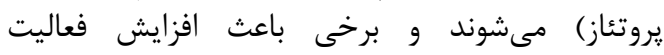

نتايج و بحث

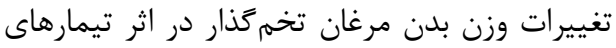

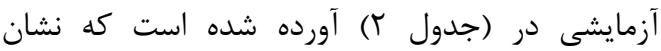

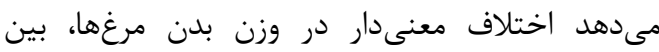

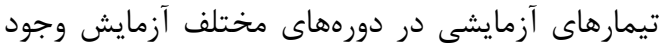

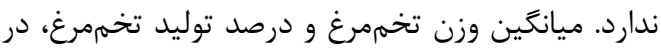

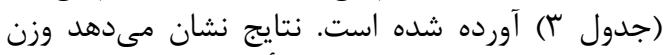

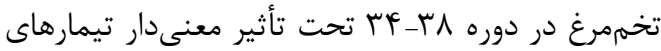

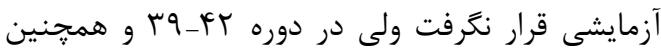

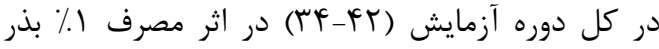

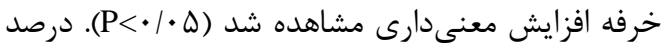

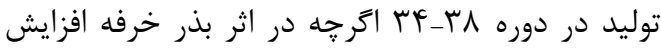

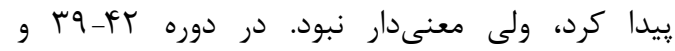

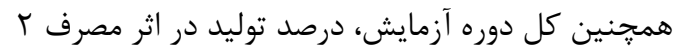

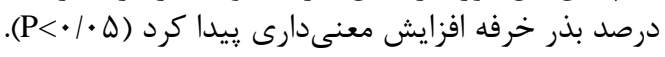

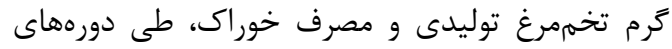

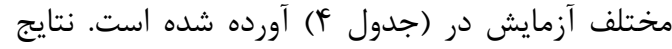

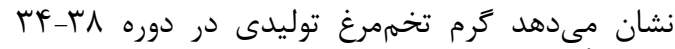

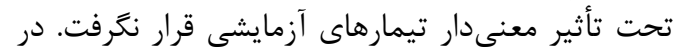

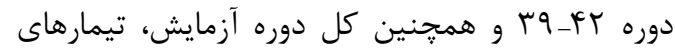

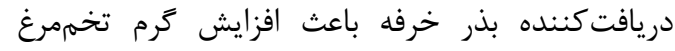

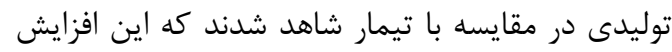

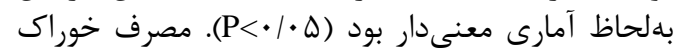




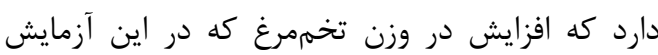

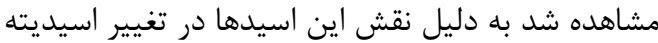

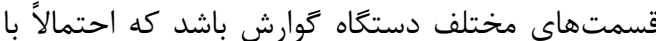

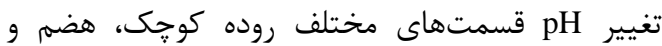

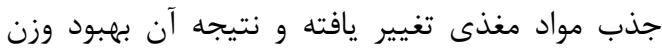

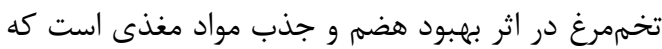

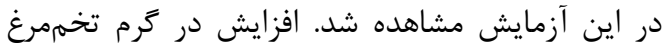

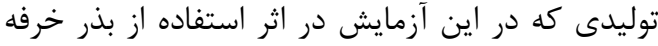

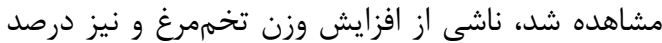

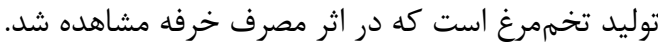

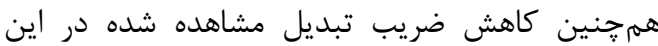

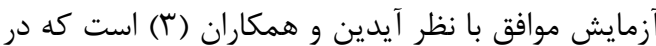

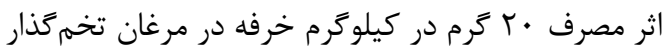

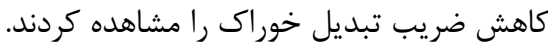

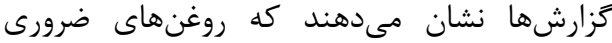

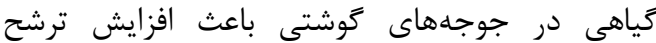

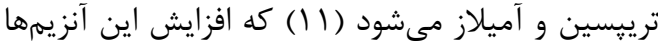

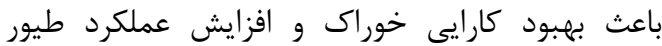

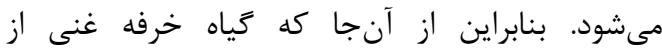

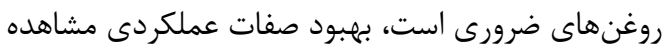

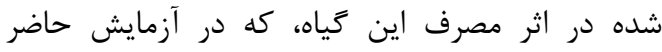

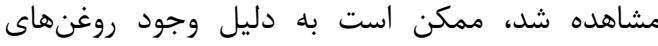

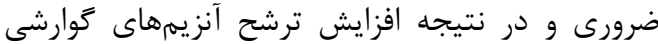

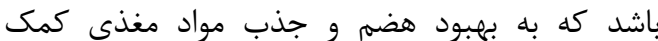

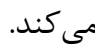

آنزيمهاى هاضم در سلولهاى موكوسى روده مى كردند

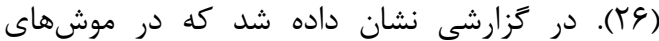

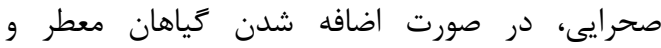

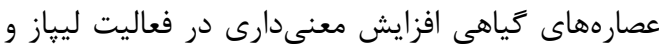

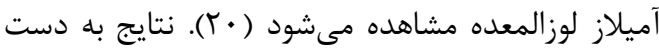

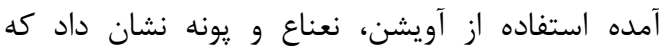

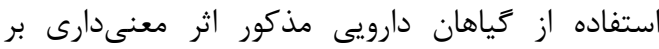

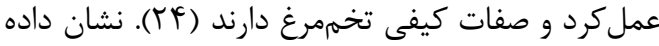

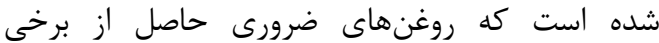

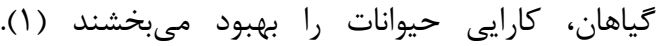

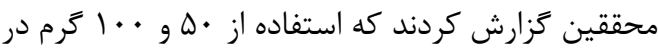

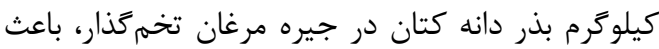

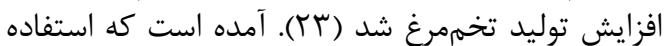

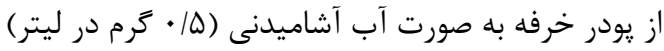

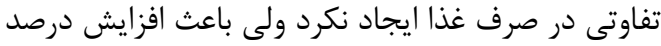

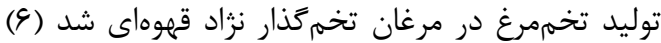

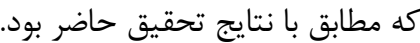

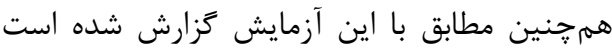

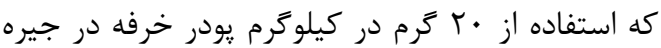

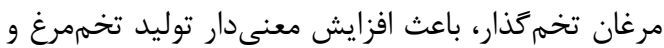

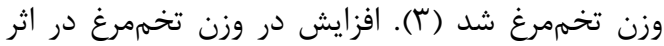

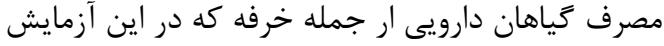

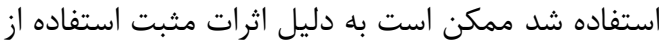

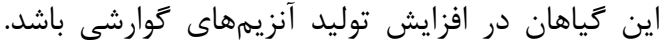

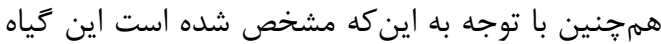

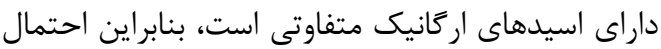

جدول r- وزن بدن مرغان تخمكذار در ابتدا و انتهاى دوره آزمايش

\begin{tabular}{|c|c|c|c|}
\hline & وزن بدن (كيلوكرم) & & \\
\hline تغييرات وزن بدن & انتهاى دوره (هفته FT) & ابتداى دوره (هفته (KF) & تيمار \\
\hline$\cdot / \cdot \vee$ & $1 / \Delta / V$ & $1 / \uparrow \Delta$ & جيره هايه (شاهد) \\
\hline$\cdot / 1 \cdot \Delta$ & 1/DSY & $1 / \& \Delta V$ & جيره حاوى |٪ خرفه \\
\hline .1 .95 & $1 / \Delta r$. & $1 / 4 \% \Delta$ & جيره حاوى ז/ خرفه \\
\hline .1 .14 &.$/ . r$. &.$/ \cdot \omega_{\Delta}$ & SEM \\
\hline . / 91 & $\cdot / \Delta \Lambda \Lambda$ & . & P-value \\
\hline
\end{tabular}

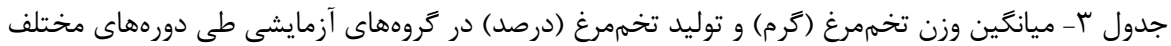

\begin{tabular}{|c|c|c|c|c|c|c|}
\hline \multirow[b]{2}{*}{ rF_Ft } & \multirow{2}{*}{ درصد توليد (./) } & \multirow[b]{2}{*}{ re-ru } & \multicolumn{3}{|c|}{ وزن تخممرغ (كرم) } & \multirow[b]{2}{*}{ تيمار } \\
\hline & & & MF_Ft & ra-kt & rF-rA & \\
\hline$\Lambda f / g)^{b}$ & $V V / \Lambda \Delta^{b}$ & $91 / \% \wedge$ & $\Delta V / 9 \varphi^{b}$ & $\Delta V / \backslash \Lambda^{b}$ & $\Delta \Lambda / V F$ & جيره پايه (شاهد) \\
\hline 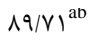 & $\Lambda F / \cdot)^{\text {ab }}$ & $9 \Delta / \varphi^{c}$ & $4 \cdot / 9 r^{\mathrm{a}}$ & $9 \cdot 199^{\mathrm{a}}$ & $91 / 19$ & جيره حاوى I. خرفه \\
\hline $9 r / \varepsilon r^{a}$ & $\Lambda \Lambda / \vee V^{a}$ & $99|0|$ & $\Delta \Lambda / \cdot \Delta^{\mathrm{D}}$ & 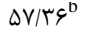 & $\Delta \Lambda / V \mu$ & جيره حاوى ז/\% خرفه \\
\hline $1 / F V T$ & I/V^G & I/V^ৎ & $\cdot / f \Delta \Lambda$ & - $/ \Delta \Delta r$ & $\cdot / \Delta \Delta r$ & SEM \\
\hline$\cdot 1 \cdot 11$ & מז •/. & $\cdot|f| f$ & $\cdot / \cdot r$ & r & .1 .99 & $\mathrm{P}$-value \\
\hline
\end{tabular}




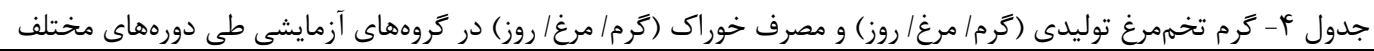

\begin{tabular}{|c|c|c|c|c|c|c|}
\hline \multirow[b]{2}{*}{ MF-FT } & مصرف خوراك (كرم/مرغ/ روز) & \multirow[b]{2}{*}{$r q-r \Lambda$} & \multicolumn{3}{|c|}{ كَرم تخممرغ توليدى (كرم/مرغ/ روز) } & \multirow[b]{2}{*}{ تيمار } \\
\hline & TQ-kT & & MF-FT & MQ-FT & $r F-r \Lambda$ & \\
\hline $91 / 91$ & $\Lambda q / 4 \Delta$ & $9 \% / V V$ & $\$ 9 / 1^{\circ}$ & $41 / 4 F^{\circ}$ & $\Delta \cdot / V V$ & جيره بايه (شاهد) \\
\hline $9 \mathrm{Q} / / \Lambda$ & $91 / 1 \mathrm{~V}$ & $9 \Delta / / \Lambda$ & $\Delta 1 / 99^{\mathrm{a}}$ & $F \vee / Q \Lambda^{a}$ & $\Delta \Delta / \Gamma r$ & جيره حاوى ا٪خرفه \\
\hline $91 / 19$ & $\Delta V / 1$. & $9 \Delta / T V$ & $\Delta 1 / .^{a}$ & 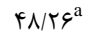 & $\Delta T / V \Delta$ & جيره حاوى r/٪ خرفه \\
\hline $1 / \pi \cdot 1$ & 1/GTT & I/GTK & - IAF. & I/Nar & I/NQT & SEM \\
\hline . $144 \Delta$ & $\cdot \mid \Delta \cdot r$ &.$/ 91$. & $.1 \cdot \cdot v \gamma$ & $.1+\psi$ & . & $P$-value \\
\hline
\end{tabular}

جدول ه- ضريب تبديل خوراك در كروههاى آزمايشى در دورههاى مختلف

\begin{tabular}{|c|c|c|c|}
\hline & ضريب تبديل (كرم/ كرم) & & \\
\hline HE - Ft & $r q-F r$ & $r r_{-}-r \Delta$ & تيمار \\
\hline$r / \cdot r^{a}$ & $r / \Lambda \Lambda^{a}$ & $1 / \wedge \Delta$ & جيره بايه (شاهد) \\
\hline $1 / \Lambda r^{\circ}$ & $1 / 9 \Delta^{\mathrm{ab}}$ & I/NT & جيره حاوى ו٪ خرفه \\
\hline$|/ N|^{0}$ & $1 / \Lambda r^{\mathrm{D}}$ & $1 / v 9$ & جيره حاوى r/٪ خرفه \\
\hline r & .1 .01 & .1 .01 & SEM \\
\hline .1 .11 & . /. t. & . /9T. & P-value \\
\hline
\end{tabular}

بر واحد هاو طى دورههاى مختلف آزمايش در (جدول

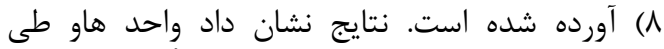

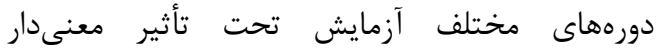
تيمارهاى آزمايشى قرار نتخرفت.

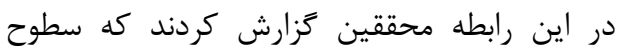

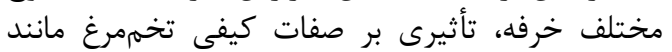

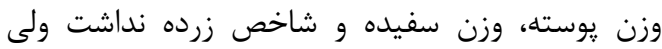

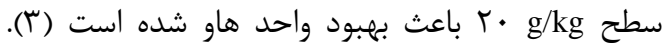

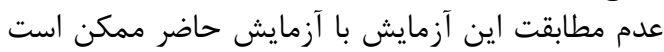

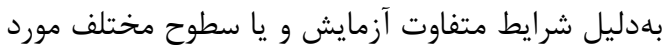

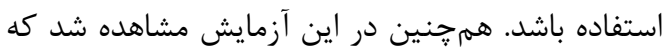

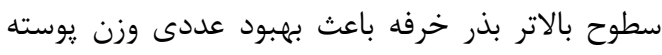

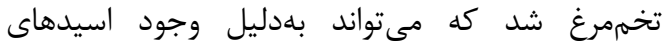

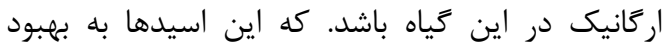

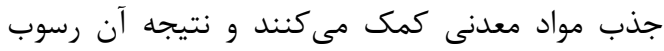

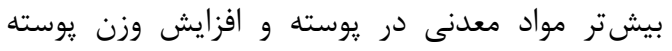

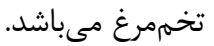

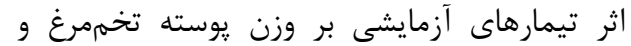

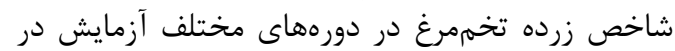

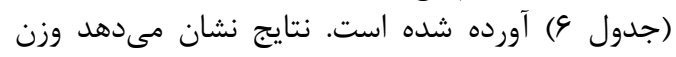

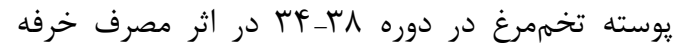

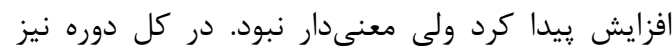

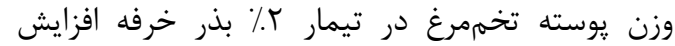

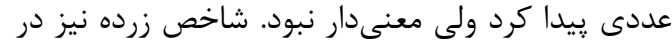

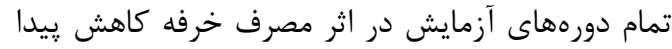

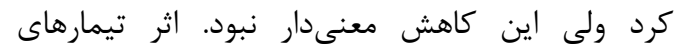

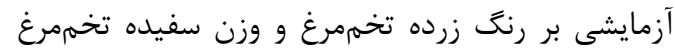

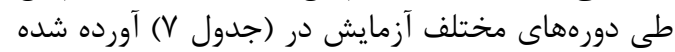

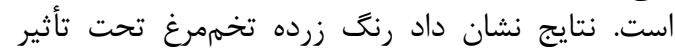

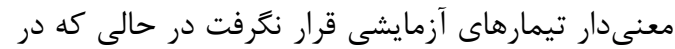

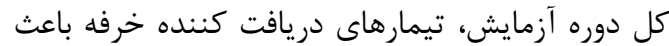

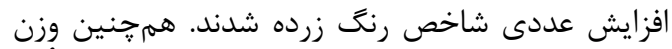

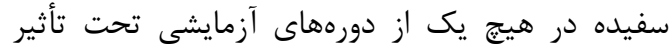
تيمارهاى آزمايشى قرار نكَرفت. اثر تيمارهاى آزمايشى

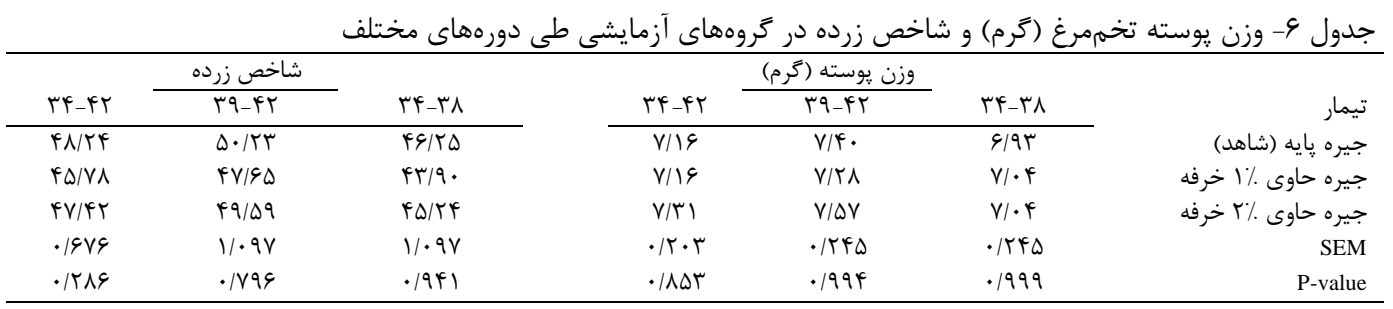


جدول V- رنخ زرده تخممرغ و وزن سفيده تخممرغ (كرم) در كروههاى آزمايشى طى دورههاى مختلف

\begin{tabular}{|c|c|c|}
\hline \multirow[b]{2}{*}{ Ty_ft } & وزن سفيده (ترم) & \multirow[b]{2}{*}{ re_r } \\
\hline & rq_fr & \\
\hline rq/f. & $F \Psi / D G$ & $r \Delta / T Y$ \\
\hline rq/АF & $F r / \cdot q$ & TV/G. \\
\hline rN/FA & FI/Tr & $r \Delta / \Delta q$ \\
\hline $1 / r q f$ & 1/Arq & $1 / 14 q$ \\
\hline • IANK & .1919 & .1991 \\
\hline
\end{tabular}

\begin{tabular}{|c|c|c|}
\hline \multirow[b]{2}{*}{ MF-FT } & رنگ زرده (عدد رش) & \multirow[b]{2}{*}{$r f-r \mu$} \\
\hline & $r q_{-} F r$ & \\
\hline $419 \mathrm{~V}$ & q/V. & $919 \pi$ \\
\hline $\mathrm{V} / \cdot \mathrm{V}$ & $\varphi / \Delta \Lambda$ & $V / \Delta \Delta$ \\
\hline G/VV & G/TK & V/rT \\
\hline.$/ 1 \Delta \Delta$ & $\cdot / \mu \cdot \Delta$ & $\cdot / r \cdot \Delta$ \\
\hline .1449 &.$/ 9 \mu \mathrm{F}$ &.$|9|$. \\
\hline
\end{tabular}

جيره پايايه (شاهد)

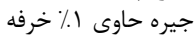
جيره حاوى بارى خرفه SEM جدول ^- اثر تيمارهاى آزمايشى بر واحد هاو طى دورههاى مختلف آزمايش

\begin{tabular}{|c|c|c|c|}
\hline & واحد هاو & & \\
\hline TF-Ft & ra-kt & rF-rA & تيمار \\
\hline TY/AT & $\Lambda 9 / r$. & $99 / 44$ & جيره پايه (شاهد) \\
\hline$\Lambda 9 / 4 \pi$ & $\Lambda V / 94$ & $9 \cdot / \mathrm{v}$ & جيره حاوى 1٪ خرفه \\
\hline $91 / \vee \Delta$ & $\Lambda 9 / \Delta \vee$ & $94 / 94$ & جيره حاوى r/ خرفه \\
\hline.$/ 9 \mu \mathrm{F}$ & 1/Q9T & 1/ब9Y & SEM \\
\hline . ITTA &.$/ q \vee \Delta$ &.$|T F|$ & P-value \\
\hline
\end{tabular}

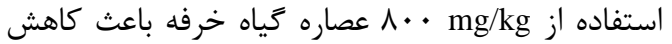

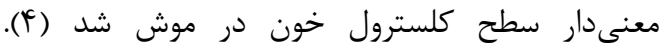

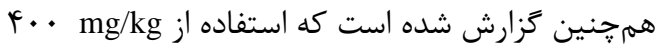

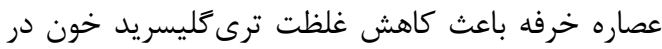

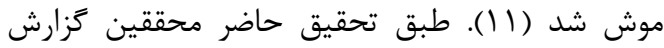

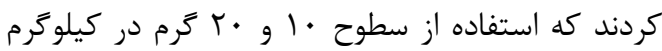

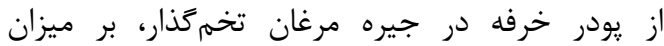

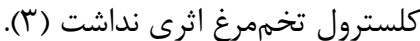
كاهش عددى ميزان كلسترول و ترى ترى تليسريد خون

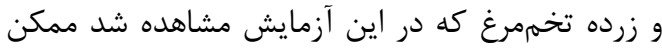

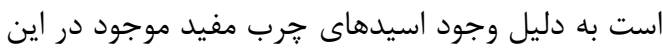

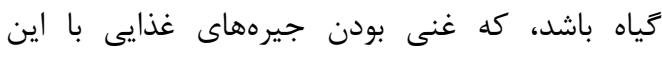

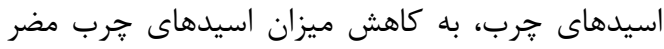

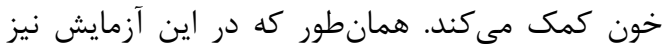

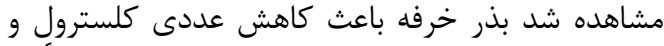
ترى كليسريد خون و زرده تخممرغ شد شد كه احتمالاًا به

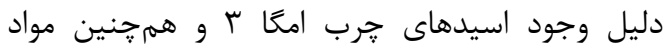

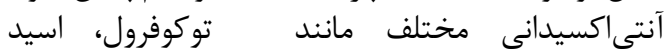

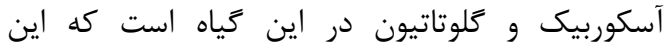
تركيبات باعث كاهش جربى هاى
اثر تيمارهاى آزمايشى بر ميزان كلسترول و و

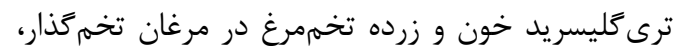

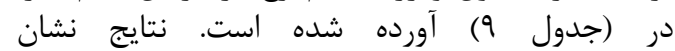

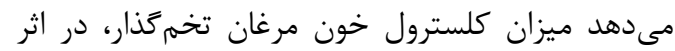

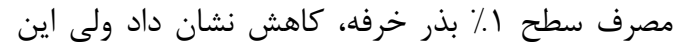

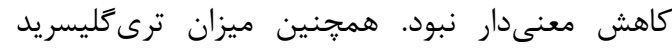

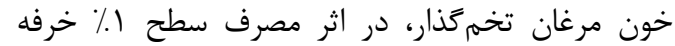

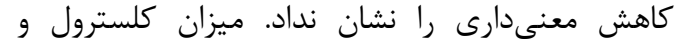

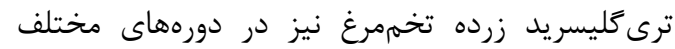

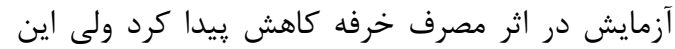

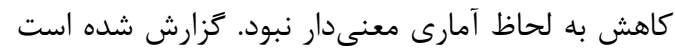

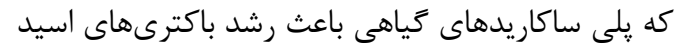

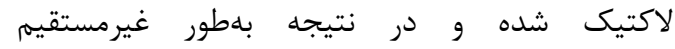

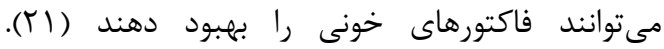
تحقيقات متعددى براى كاهش كلسترول وترنى

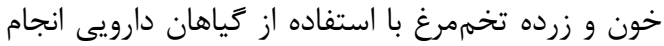

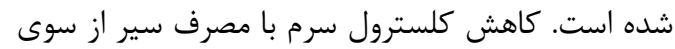

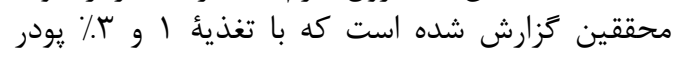

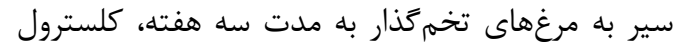

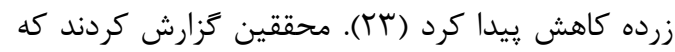

\begin{tabular}{|c|c|c|c|c|c|c|}
\hline \multicolumn{4}{|c|}{ مهمرغ در مرغان تخمكخذار } & \multicolumn{2}{|c|}{ خون (mg/dl) } & \multirow[b]{2}{*}{ تيمار } \\
\hline ترى دليسريد & ترى دليسريد & كلسترول & كلسترول & ترى كليسريد & كلسترول & \\
\hline $10 \cdot / \cdot$ & $\mid r r / \cdot \cdot$ & $\mid r / \cdots$ & $1 \cdot / \omega$ & TMTT/Q. & $\mid \wedge \Delta / \cdot \cdot$ & جيره پِايه (شاهد) \\
\hline $\mid r F / 99$ & TrQTr & $9 / 99$ & 1.1. & $19 r F / 0$. & $\mid F F / V D$ & جيره حاوى 1٪ خرفه \\
\hline $\mid r q / r \Delta$ & $1.9 / .$. & $1 \cdot 1$. & $q / v \Delta$ & $r T \Delta F / .$. & $\mid \wedge \mathrm{V} / .$. & جيره حاوى r/ خرفه \\
\hline$r \cdot / \Lambda F \Delta$ & $19 / \pi \cdot 9$ & $1 / 1 \mathrm{~V}$. & $1 / 9 \wedge \mathrm{V}$ & $r<q / .$. & $|1 / 99|$ & SEM \\
\hline - /19V & $\cdot|q 4|$ & $\cdot / T V F$ &.$/ 9 \Delta V$ & $.19 \cdot 9$ & $\cdot / T F$ & P-value \\
\hline
\end{tabular}

توجه به نتايج متفاوت به دست آمده در مورد سطوح

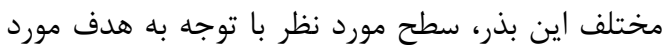

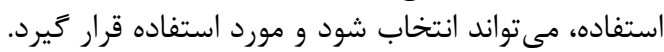

با توجه به نتايج به دست آمده از اين آزمايش، به نظر

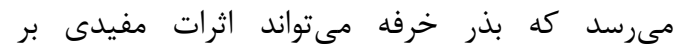

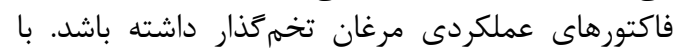


1. Alcicek A. M. Bozkurt and M. Cabuk 2003. The effect of an essential oil ombination derived from selected herbs growing wild in Turkey on broiler performance. South African Journal of Animal Science, 33: 89-94.

2. AOAC. 1990. Official Methods of Analysis of the AOAC.15th edition. Arlington, VA. pp: 69-74.

3. Aydin, R. and I. Dogan. 2010. Fatty acid profile and cholesterol content of egg yolk from chickens fed diets supplemented with purslane (Portulaca oleracea L.). Journal of the Science of Food and Agriculture, 90: $1759-1763$.

4. Changiziashtiyani, S., A. Zareei, S. Taheri, F. Rasekh and V. Ramazani. 2012. Effect of alcoholic extract of purslane (Portulaca Oleracea) on hypercholesterolaemia in rats. Zahedan Journal of Research in Medical Sciences, Print the following.

5. Craig, W.J. 1999. Health-promoting properties of common herbs. The American Journal of Clinical Nutrition, 70: 491-499.

6. Dougnon, T.J., C. Anago and M.N. Assogba. 2012. Effects of portulaca oleracea linn and extra egg® in eggs'stimulation in ISA brown layer. International Journal of Science and Nature, 3: 69-72.

7. Ezekwe, M.O., T.R. Omara-Alwala and T. Membrahtu. 1999. Nutritive characterization of purslane accessions as influenced by planting date. Plant Foods for Human Nutrition, 54: 183-91.

8. Farkhoy, M., T. Khalighysigarody and F. Niknafas. 1984. Poultry breeding guide. Coasar Press, pp: 150266.

9. Funk, E.M. 1948. The relation of yolk index determined in natural position to the yolk index as determined after separating the yolk from the albumen. Poultry Science, 27: 367.

10. Gatreh-Samani, K., B. Khalili, M. Rafieian and M.T. Moradi. 2011. Purslane (Portulaca oleracea) effects on serum paraoxanase-1 activity Persian. The "Journal of Shahrekord University of Medical Sciences, 13: 9-16.

11. Janz, J.A.M., P.C.H. Morel, B.H.P. Wilkinson and R.W. Purchas. 2007. Preliminary investigation of the effects of low-level dietary inclusion of fragrant essential oils and oleoresins on pig performance and pork quality. Meat Science, 75: 350-355.

12. Jingrong, L.U., H.E. Turong and R. Putheti. 2009. Compounds of Purslane extracts and effects of antikinetic fatigue. Journal of Medicinal Plants Research, 3: 506-510.

13. Liu, L., P. Howe, Y.F. Zhou, Z.Q. Xu, C. Hocart and R. Zhan. 2000. Fatty acids and beta- carotene in australian purslane (portulaca oleracea) varieties. Journal of Chromatography, Sep 29, 893: 127-132.

14. Luhman, C.M., B.G. Miller and D.C. Beitz. 1990. The effect of feeding lovastatin and colestipol on production and cholesterol content of eggs .Poultry Science, 69: 852-855.

15. Miladi-Gorgi, H., A.A. Vafaei, A.A. Taherian and T. Vaezi. 2009. The effects of aqueous extracts of Purtulaca oleracea on withdrawal syndrome in mice Iranian Journal of Medicinal Plants, 8: 51-57.

16. Miladi-Gorii, H., A.A. Vafaei and A. Bageri. 2011. To investigate the effect of (Portulaca oleracea L.) and Melissa officinalis L. extract on sleeping time in mice] Iranian Journal of Medicinal Plants, 10: 95-101 (In Persian).

17. Nobakht, A. 2009. Effects of herbs thyme, mint and oregano on performance and egg quality of laying hens. Proceedings of the conference on the development of industry of medicinal plants, $11 \mathrm{pp}$.

18. North, M.O. 1984. Commercial chicken production manual. $3^{\text {rd }}$ Edition. $291 \mathrm{pp}$.

19. Omara-Alwala, T.R., T. Mebrahtu, D.E. Prior and M.O. Ezekwe. 2007. Omega-three fatty acids in purslane (Portulaca oleracea) tissues. Journal of the American Oil Chemist Society, 68: 198-199.

20. Rao, R.R., K. Platel and K. Srinivasan. 2003. In vitro influence of spices and spice-active principles on digestive enzymes of rat pancreas and small intestine. The Journal of Nahrung, 47: 408-412.

21. Sakine, Y., E. Ebru, Z. Reisli and Y. Suzan. 2006. Effect of garlic powder on the performance, egg traits and blood parameters of laying hens. Journal of Food Science, 86: 1336-1339.

22. Savage, T.F., P.F. Cotter and E.I. Zakrzewska. 1996. The effect of feeding mannan oligosaccharide on immunoglobulins, plasma Ig G and bile Ig A, of wrolstad MW mal turkeys. Poultry Science, 75: 143-152.

23. Scheideler, S.E. and G.W. Froning. 1996. The combined influence of dietary flax seed variety, level, form and storage conditions on egg production and composition among vitamin E-supplemented hens. Poultry Science, 75: 1221-1226.

24. Sharma, R.K., R.A. Singh, R.N. Pal and C.K. Aggarwal. 1979. Cholesterol content of chicken eggs as affected by feeding garlic, sarpagandha and nicotinic acid. Haryana Agricultural University Journal of Research, 9: 263-265.

25. Song, J., H. Fan, Y. Zhao, Y. Jia, X. Du and B. Wang. 2008. Effect of salinity on germination, seedling emergence, seedling growth and ion accumulation of a euhalophyte Suaeda salsa in an inter-tidal zone and on saline inland. Aquatic Botany, 88: 331-337.

26. Srinivasan, K. 2005. Spices as influencers of body metabolism. Food Research International. 38: 77-86.

27. Zargari, A. 2001. Medicinal herbs. Institute is publishing and printing of University of Tehran. $3^{\text {rd }}$ Edition. I: $233-241$. 


\title{
Effect of Different Levels of Purslane Seed (Portulaca Oleracea) on Performance and Some Egg Characteristics in Laying Hens
}

\author{
Mosayeb Shallai ${ }^{1}$ and Seyyed Mohammad Hosseini ${ }^{2}$ \\ 1- Gradated M.Sc., Brigand University (Corresponding author: mosayeb_shalaey@yahoo.com) \\ 2- Associate Professor, Brigand University \\ Received: August 1, $2013 \quad$ Accepted: April 6, 2014
}

\begin{abstract}
This experiment was conducted to evaluate the effect of different levels of purslane seeds on the production performance, some egg characteristics and blood and yolk cholesterol and triglyceride levels, in different periods of laying hens. The experiment was done on hay-line strain (w36) from 32 to 42 weeks using a completely randomized design with 3 treatments, 4 replicate and 8 hens in each replicate. The experimental diets were, 1- basal diet (Control), 2 -Treatment containing $1 \%$ purslane seed and 3-Treatment containing $2 \%$ purslane seed. The results showed that egg weights were significantly increased in the treatment having $1 \%$ purslane seed $(\mathrm{P}<0.05)$. Percent of the egg production in the hens fed $2 \%$ purslane seed was significantly increased compared to the control group $(\mathrm{P}<0.05)$. The egg mass in the groups receiving purslane seed showed a significant increase $(\mathrm{P}<0.05)$. The feed conversion ratio was also improved by consumption of purslane seeds $(\mathrm{P}<0.05)$. Egg characteristics were not significantly affected by dietary treatments. Consequently the level of $1 \%$ purslane seed have positive effect on egg weight and level of $2 \%$ purslane seed improves egg mass and feed conversion ratio in laying hens.
\end{abstract}

Keywords: Egg characteristics, Laying hens, Performance, Purslane seed, Yolk cholesterol 